\title{
Effect of Buffer Quality on the Performance of InAs/AlSb Heterostructure Backward Tunneling Diode
}

\author{
Huan Zhao ${ }^{1}$, Parisa Yadranjee Aghdam ${ }^{2}$, Zonghe Lai $^{3}$ \\ ${ }^{1}$ Terahertz and Millimeter Wave Laboratory, ${ }^{2}$ Group for Advanced Reciever Development, ${ }^{3}$ Nanofabrication Laboratory \\ Department of Microtechnology and Nanoscience, Chalmers University of Technology, SE-41296, Sweden
}

\begin{abstract}
InAs/AlSb Heterostructure Backward Tunneling Diodes (HBTDs) were grown on semi-insulating GaAs (100) substrate using molecular beam epitaxy (MBE). The current-voltage characteristics of the InAs/AISb HBTDs, both at room temperatures (RT) and cryogenic temperatures, have been studied as a function of the InAs buffer thickness. It has been found that a thicker InAs buffer doesn't improve the surface roughness but decreases the threading dislocation (TD) density, and thus results in a higher curvature coefficient in the current-voltage characteristics near zero-bias.
\end{abstract}

\section{INTRODUCTION}

$\mathrm{M}$ ILLIMETER wave and submillimeter wave detection and imaging have attracted considerable interest due to its potential in applications such as security, defense, and medical diagnosis. For such sensing and imaging systems, highly sensitive and compact solid-state detectors are the key components. Among a number of millimeter wave detection techniques, InAs/AlSb heterostructure backward tunneling diode (HBTD) has been found to be one of the most promising candidates for direct detection in this wavelength range. These diodes exhibit especially high curvature in the current-voltage characteristic that produces the rectification without bias. A record matched sensitivity of $50 \mathrm{kV} / \mathrm{W}$ and noise equivalent power of $0.18 \mathrm{pW} / \mathrm{Hz}^{1 / 2}$ have been demonstrated at $94 \mathrm{GHz}$ at room temperature (RT) [1], which challenges other existing RT detectors in this wavelength range.

However, the epitaxy growth of these device structures is challenging, mainly due to the lack of a lattice matched semi-insulating substrate. Thus, much of the growth is carried out on GaAs substrate, which has a large lattice mismatch (7\%) respect to InAs. Since the tunneling barriers of the InAs/AlSb HBTDs are usually very thin $(1-3 \mathrm{~nm})$, interface roughness or fluctuation in tunneling barrier thickness are expected to be important [2]. On the other hand, dislocations especially the threading dislocations (TDs) are usually detrimental to device performances. However, up to now few has reported on how the material growth and the buffer quality effect on the performance of the InAs/AlSb HBTDs.

In this paper, the current-voltage characteristics of InAs/AlSb HBTDs, grown by molecular beam epitaxy (MBE) on GaAs substrate, are reported as a function of the InAs buffer layer thickness. The effect of the buffer quality on the performance of the InAs/AlSb HBTDs will be discussed.

\section{EXPERIMENT}

All samples in this study were grown by a Riber compact $\mathrm{C} 21$ MBE system. Four HBTD samples were grown on GaAs semi-insulating (100) substrates through InAs buffers with different thicknesses, i.e. $0 \mu \mathrm{m}, 0.5 \mu \mathrm{m}, 1 \mu \mathrm{m}$, and $1.5 \mu \mathrm{m}$, see Fig.1. Two InAs buffers samples with $1 \mu \mathrm{m}$ and $2 \mu \mathrm{m}$ thicknesses were grown to check the surface morphology at the InAs/GaSb interface (including the $0.5 \mu \mathrm{m}$ thick InAs contact layer). The samples were characterized by cross-sectional Transmission Electron Microscopy (XTEM) or Atomic Force Microscopy (AFM). Test diodes with $60 \times 60 \mu \mathrm{m}^{2}$ large cathode size were fabricated. The current-voltage characteristics of the HBTDs were performed both at RT and cryogenic temperature.

\begin{tabular}{|lc|}
\hline $\mathrm{n}++$ InAs & $1000 \AA$ \\
\hline $\mathrm{n}$ InAs cathode & $500 \AA$ \\
\hline AlSb & $30 \AA$ \\
\hline $\mathrm{Al}_{0.12} \mathrm{GaSb}$ & $150 \AA$ \\
\hline $\mathrm{p}++\mathrm{GaSb}$ anode & $300 \AA$ \\
\hline $\mathrm{n}++$ InAs & $5000 \AA$ \\
\hline InAs buffer & $0-1.5 \mu \mathrm{m}$ \\
\hline GaAs semi-insulating $(100)$ substrate \\
\hline
\end{tabular}

Fig.1: Epitaxy structures of the HBTDs grown InAs buffers with different thicknesses.

\section{RESULTS AND DISCUSSIONS}

Fig. 2 shows the I-V curves of the HBTDs grown on InAs buffers with different thicknesses, measured at both RT and $7 \mathrm{~K}$. At RT as the InAs buffer thickness increases, the diode shows a larger current at reverse bias $(<0 \mathrm{~V})$, a smaller current at low forward bias $(<\sim 0.37 \mathrm{~V})$ and a larger current at high forward bias $(>\sim 0.37 \mathrm{~V})$. The curvature coefficient, defined as $\left(d^{2} I / d V^{2}\right) /(d I / d V)$, increases from $-6 \mathrm{~V}^{-1}$ to $-17 \mathrm{~V}^{-1}$ as the buffer thickness increases from $0 \mu \mathrm{m}$ to $1.5 \mu \mathrm{m}$. By comparing the I-V curves of the same diode at RT and $7 \mathrm{~K}$, the difference in currents at low forward bias becomes negligible between the samples.
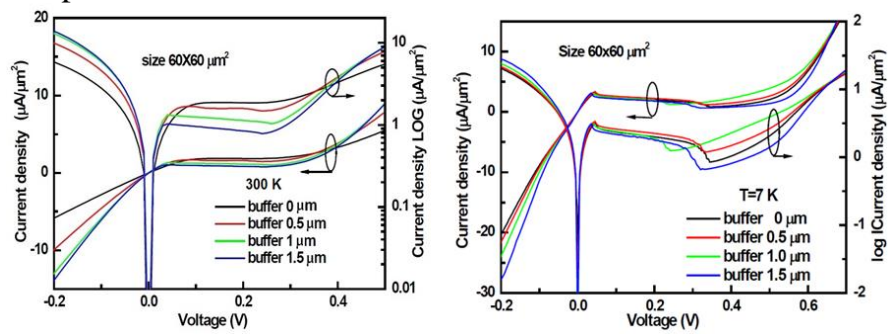

Fig.2: I-V curves of the HBTDs grown on InAs bulk layer with different thicknesses, measured at RT and $7 \mathrm{~K}$. 
To understand the effect of buffer quality on the performance of the diode performance, XTEM was performed. Fig. 3 shows the XTEM images of the full HBTD structures grown on InAs buffers with different thicknesses. Obviously the TD density decreases as the thickness of the InAs buffer layer increases [3]. A great reduction of the TD density is observed in the sample grown on $1.5 \mu \mathrm{m}$ thick InAs buffer. Zoom-in XTEM images of the active region of the HBTDs are shown in Fig.4. The tiny bright lines indicate the AlSb tunneling barriers. No obvious difference in thickness fluctuation of the AlSb barriers is observed between the samples. A further AFM measurement, see Fig.5, shows that the root mean square (RMS) roughness for the $1 \mu \mathrm{m}$ thick and the $2 \mu \mathrm{m}$ thick InAs buffers is $2.7 \mathrm{~nm}$ and 3 $\mathrm{nm}$, respectively.

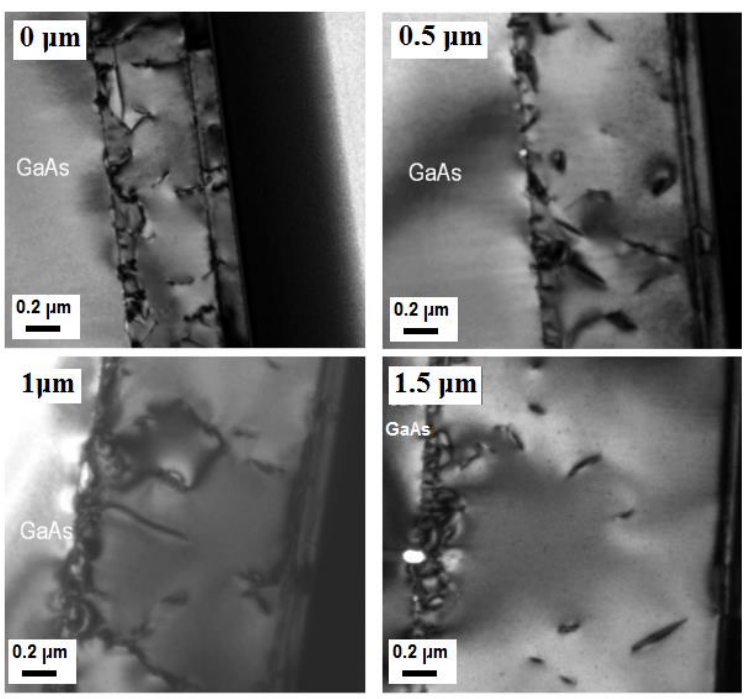

Fig.3: XTEM images of the full HBTD structures grown on InAs buffers with different thicknesses.
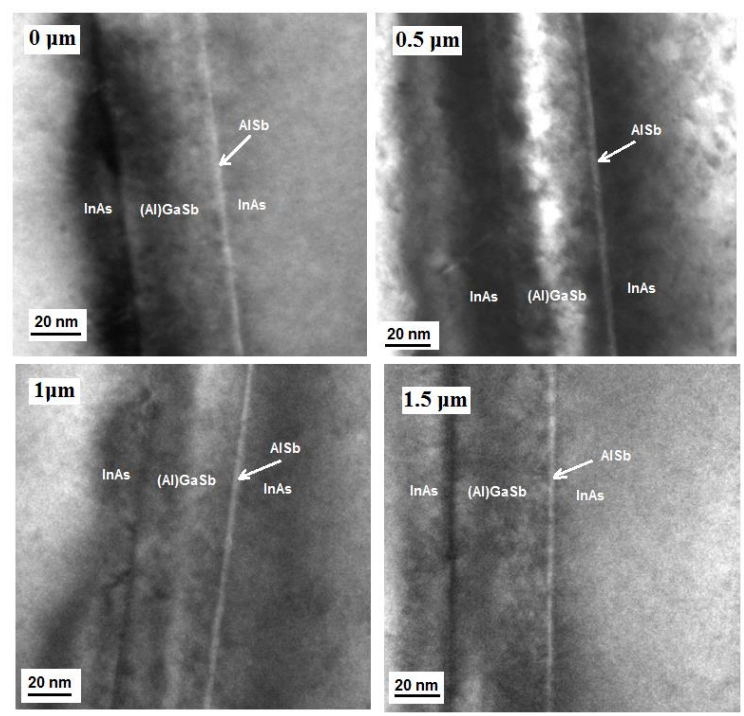

Fig.4: X-TEM images of the active regions of the HBTDs grown on InAs buffers with different thicknesses, revealing the InAs/AlSb/(Al) $\mathrm{GaSb}$ heterostructures.
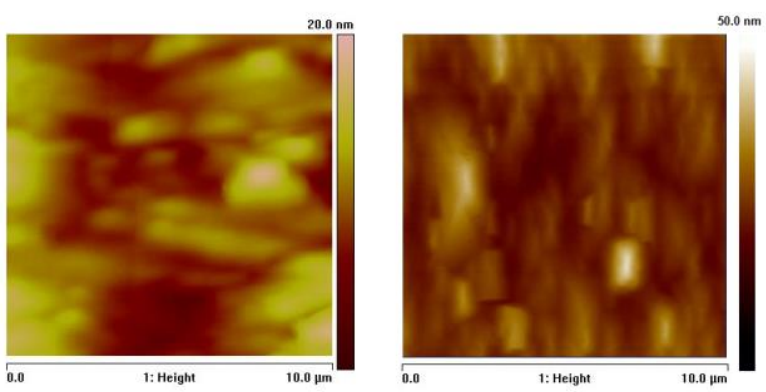

Fig.5: $10 \times 10 \mu \mathrm{m}^{2}$ AFM images for the $0.5 \mu \mathrm{m}$ (left) and the $1 \mu \mathrm{m}$ thick (right) InAs buffer.

Therefore, a thicker InAs buffer doesn't improve the surface roughness but decreases the TD density. The difference in the I-V characteristics of the HBTDs is mainly attributed to the different TD densities in the InAs buffers. For diodes grown on thinner buffers, the lower current at reverse bias and high forward bias is due to the high resistive $p-n$ junctions, and the higher current at low forward current (excess current) is due to the trap-assisted tunneling. Both the high resistivity and the trap-assisted tunneling are attributed to the high TD densities. As the phonon energy required for trap-assisted tunneling is more probable to be obtained at RT rather than at cryogenic temperature, therefore the difference of the currents at low forward bias becomes negligible at cryogenic temperatures. Other changes in the I-V curves at cryogenic temperature compared with RT are because of changes of the band gap and resistivity of the materials with temperatures.

\section{CONCLUSIONS}

InAs/AlSb HBTDs were grown by MBE on GaAs substrates. The current-voltage characteristics of the diodes are reported as a function of the InAs buffer layer thickness. The RT curvature coefficient increases as the InAs buffer thickness increases. According the AFM and the XTEM measurements, a thicker InAs buffer doesn't improve the surface roughness but decreases the TD density. The difference in the I-V characteristics of the HBTDs grown on different InAs buffers is mainly attributed to the different TD densities.

\section{CONCLUSIONS}

This work is supported by the Swedish research council (VR).

\section{REFERENCES}

[1]. Z. Zhang, R.Rajavel, P.Deelman, P.Fay, "Backward diode millimeter-wave detectors with $0.18 \mathrm{pW} / \mathrm{Hz} 1 / 2$ noise equivalent power", Microw. Wireless. Comp. Lett., Vol.21, pp.267-269, 2011.

[2]. P. Y. Aghdam, H. Zhao, "Modeling of Sb-heterostructure backward diode for millimeter- and submillimeter-wave detection", physcs status solidi (c), Vol.10, pp. 777-781, 2013.

[3]. A. E. Romanov, W. Pompe, S. Mathis, G. E. Beltz, and J. S. Speck, "Threading dislocation reduction in strained layers," Journal of Applied Physics, vol. 85, pp. 182-192, Jan. 1999. 